\title{
MicroRNA-149 contributes to scarless wound healing by attenuating inflammatory response
}

\author{
HONGXIN LANG ${ }^{1}$, FENG ZHAO ${ }^{1}$, TAO ZHANG ${ }^{1}$, XIAOYU LIU ${ }^{2}$, \\ ZHE WANG ${ }^{3}$, RUI WANG ${ }^{1}$, PING SHI ${ }^{4 *}$ and XINING PANG ${ }^{*}$

\begin{abstract}
${ }^{1}$ Department of Stem Cells and Regenerative Medicine, Key Laboratory of Cell Biology, Ministry of Public Health and Key Laboratory of Medical Cell Biology, Ministry of Education, China Medical University, Shenyang, Liaoning 110004; ${ }^{3}$ Department of Pathology, Shengjing Hospital of China Medical University; ${ }^{4}$ Department of General Practice, First Hospital of China Medical University, Shenyang, Liaoning 110001, P.R. China
\end{abstract} \\ Liaoning 110013; ${ }^{2}$ Department of Assisted Reproduction, Shengjing Hospital of China Medical University, Shenyang,
}

Received December 5, 2016; Accepted May 31, 2017

DOI: $10.3892 / \mathrm{mmr} .2017 .6796$

\begin{abstract}
A fibrotic or pathological scar is an undesired consequence of skin wound healing and may trigger a series of problems. An attenuated inflammatory response is a significant characteristic of fetal skin wound healing, which can contribute to the scarless healing of fetal skin. According to deep sequencing data, microRNA-149 (miR-149) expression was increased in mid-gestational compared with that in late-gestational fetal skin keratinocytes. It was demonstrated that overexpression of miR-149 in HaCaT cells can downregulate the expression of pro-inflammatory cytokines interleukin (IL)-1 $\alpha$, IL-1 $\beta$, and IL- 6 at basal levels and in inflammatory conditions. Furthermore, miR-149 was revealed to indirectly accelerate transforming growth factor- $\beta 3$ and collagen type III expression in fibroblasts, which are essential cells in extracellular matrix remodeling. In a rat skin wound model, miR-149 improved the quality of the arrangement of collagen bundles and reduced inflammatory cell infiltration during skin wound healing. These results indicate that miR-149 may be a potential regulator in improving the quality of skin wound healing.
\end{abstract}

Correspondence to: Professor Ping Shi, Department of General Practice, First Hospital of China Medical University, 155 North Nanjing Street, Shenyang, Liaoning 110001, P.R. China

E-mail: 13998309428@163.com

Professor Xining Pang, Department of Stem Cells and Regenerative Medicine, Key Laboratory of Cell Biology, Ministry of Public Health and Key Laboratory of Medical Cell Biology, Ministry of Education, China Medical University, 77 Puhe Street, Shenyang, Liaoning 110013, P.R. China

E-mail: pangxining@126.com

${ }^{*}$ Contributed equally

Key words: inflammation, skin wound healing, scar formation, the synthesis and degradation of extracellular matrix

\section{Introduction}

Skin wound healing is a sophisticated and sequential physiological process, with three independent and simultaneously overlapping stages, namely inflammation, proliferation, and remolding. A skin wound heals depending on well-orchestrated biological processes, including the production of cytokines, synthesis and degradation of the extracellular matrix, cell lineages, specifics of the wound, and age of the patients (1). In adult skin wound healing, wounds are usually accompanied by scar formation, with a mature scar forming a narrow, flat, and hypo-pigmented appearance, which may depress with time (2). Pathological or fibrotic scars are caused by dysregulation of the wound healing mainly related to unregulated inflammation, abnormal cell proliferation, excessive collagen deposition, abnormal cytokine production, and imbalance between synthesis and degradation of the extracellular matrix (3). Pathological or fibrotic scars can lead to serious problems, such as physiological dysfunction, a defective appearance, and an economic burden. Fetal skin is prone to heal without scaring in a given period, due to intrinsic abilities, such as minimal inflammation, special fibroblast phenotypes, and certain gene expression profiles. To date, researchers have made several efforts to find effective therapies to heal aberrant scar formation in clinical cases.

Inflammation is a crucial part of the healing process $(4,5)$, defending against damage from pathogenic microorganisms and regulating the production of cytokines and angiogenesis. Diverse inflammatory cytokines and cells were involved in wound healing process. Immediately after injury, neutrophils are the first cells to infiltrate the wound site and help to destroy foreign particles. Later, macrophages are attracted to the wound site to phagocytize bacteria and tissue debris (6), however, an excessive macrophage presence can stimulate scarring. Some pro-inflammatory cytokines, such as IL-1 (7), IL-6, IL-8 (8), IL-17 (9), IL-18 (10), and IL-33 (11) have been reported to participate in fibrosis.

MicroRNAs (miRNAs or miRs) are a class of endogenous, highly conserved non-coding small RNAs that regulate most eukaryotic protein-coding genes. Their 'seed regions' (located 
on miRNA nucleotides $2-8$ from the 5 ' region) bind to the reverse complement of the 3'-untranslated region (3'-UTR) of the target mRNA, resulting in its degradation or repressed translation. miRNAs participate in development, disease progression, cell proliferation, differentiation, apoptosis, and many other processes. miRNAs are also involved in inflammation, scar formation, and angiogenesis during skin wound healing.

miR-149 has an important regulatory role in many fields, it has been reported to regulate AKT1/mTOR and TGF- $\beta$ /SMAD signaling pathway (12-14). In addition, miR-149 is closely associated with inflammation. miR-149 can regulate TNF- $\alpha$, IL- 6 , and iNOS, in order to regulate the pathogenesis of osteoarthritis (15) and endothelial dysfunction (16). As reported in the current study, miR-149 is downregulated in the inflammatory phase in skin tissues at $24 \mathrm{~h}$ compared to $0 \mathrm{~h}$ after wounding (17), and it shows a lower expression in scars compared to normal skin tissue (18).

In a previous study, we performed small RNA next-generation sequencing to uncover the dynamic changes between miRNAs in mid- and late-gestational fetal skin keratinocytes (19). We found that many miRNAs have different expression patterns between mid- and late-gestation. In total, 88 known miRNAs exhibit more than a two-fold change in expression in fetal keratinocytes, fifteen of which are significantly upregulated, whereas the others significantly downregulated. Multiple miRNAs regulate the AKT/mTOR, TGF- $\beta /$ SMAD, and NF- $\mathrm{B}$ pathways, which affect the skin wound healing process. We also found that several interleukins are downregulated in mid-gestation fetal keratinocytes, as compared to those in late-gestation. We focused on miR-149, which is upregulated in mid-gestation fetal keratinocytes, and whose predicted target mRNAs are IL- $1 \alpha$, IL- $1 \beta$ and IL- 6 . We explored the anti-inflammatory and anti-scarring effects of miR-149 in the skin wound healing process through regulation of the expression of IL- $1 \alpha$, IL-1 $\beta$, and IL- 6 .

\section{Materials and methods}

Cell culture and treatments. The human immortal keratinocyte cell line, HaCaT cells and human dermal fibroblasts (both cells were obtained from the Department of Stem Cell and Regenerative Medicine) used in this study were cultured in Dulbecco's Modified Eagle's Medium (DMEM)-High Glucose (HyClone, Logan, UT, USA) with $10 \%$ fetal bovine serum (FBS; Invitrogen Life Technologies, Carlsbad, CA, USA) at $37^{\circ} \mathrm{C}$ and $5 \% \mathrm{CO}_{2}$.

$\mathrm{HaCaT}$ cells were transfected with miR-149 mimic or a negative control (50 mM; GenePharma, Shanghai, China) using the Lipofectamine ${ }^{\circledR} 2000$ reagent (Invitrogen Life Technologies) according to the manufacturer's protocol.

To analyze the effect of miR-149 on inflammatory cytokines, treatments with and without TNF- $\alpha$ were used to reflect the inflammatory and basal environments, respectively. $\mathrm{HaCaT}$ cells were transfected with miR-149 mimic or a negative control, treated $48 \mathrm{~h}$ later with or without TNF- $\alpha(20 \mathrm{ng} / \mathrm{ml}$; Peprotech, Inc., Rocky Hill, NJ, USA), and then cultured in an incubator for another $24 \mathrm{~h}$.

The dynamic expression of miR-149 between mid- and late-gestational fetal skin keratinocytes was measured by next-generation sequencing (19).
Co-culture assay. To explore how HaCaT cells transfected with the miRNA mimic or a negative control affected the behavior of dermal fibroblasts, $\mathrm{HaCaT}$ cells and fibroblasts were co-cultured in transwell polyester membrane inserts with a $0.4 \mu \mathrm{m}$ pore size (CoStar, Corning, NY, USA) for $48 \mathrm{~h}$. Then the fibroblasts were harvested to detect the mRNA and protein expression.

Migration assay. The migration of HaCaT cells from serum-free media to standard culture media was measured in 24-well transwell culture inserts with an $8.0 \mu \mathrm{m}$ pore size (CoStar), as described previously (20). HaCaT cells were hematoxylin-eosin stained (Solarbio, Beijing, China) at $36 \mathrm{~h}$ and counted from sixteen fields per well randomly.

An in vitro scratch assay was used to detect the migration of the HaCaT cells. After transfection for $48 \mathrm{~h}$, a $200 \mu \mathrm{l}$ tip was used to make a scratch. The cells were washed with PBS to discard the cell debris, and then photographed under a microscope at 0,24 and $36 \mathrm{~h}$.

$R T$-qPCR. We used RT-qPCR to detect gene expression. To detect microRNAs, RNAs were prepared from cells using the mirVana miRNA Isolation kit (Ambion Life Technologies, Carlsbad, CA, USA), and was added a poly (A) tail to the RNA transcripts by the Poly (A) Tailing kit (Ambion Life Technologies). Reverse transcription was performed with an RT Reagent kit with gDNA Eraser (Takara Bio, Inc., Otsu, Japan), and RT-qPCR was performed using a SYBR Premix Ex Taq II kit (Takara Bio, Inc.) on the 7500 Real-Time PCR system (Applied Biosystems Life Technologies, Foster City, CA, USA). To detect mRNA expression, total RNA was prepared using the TRIzol (Invitrogen Life Technologies) reagent, and reverse transcription and RT-qPCR were performed as above. The RT-qPCR reaction conditions were $95^{\circ} \mathrm{C}$ for $30 \mathrm{sec}$, followed by 40 cycles of $95^{\circ} \mathrm{C}$ for $5 \mathrm{sec}$ and $60^{\circ} \mathrm{C}$ for 34 sec. U6 and GAPDH were used as endogenous reference genes. The primers used in RT-qPCR were shown in Table I. The $2^{-\Delta \Delta \mathrm{Cq}}$ method was used to calculate the change in gene expression.

Elisa analysis. To detect secretory protein levels affected by miR-149, cell culture supernatants were collected from $\mathrm{HaCaT}$ cells transfected with miR-149 mimic or a negative control and the protein expression of IL- $1 \alpha$, IL- $1 \beta$, and IL- 6 were measured using ELISA kits (R\&D Systems, Inc., Minneapolis, $\mathrm{MN}, \mathrm{USA}$ ) according to the manufacturer's instruction.

Validation of target mRNAs. The target genes of miR-149 were predicted on the website TargetScan (http://www.targetscan .org/) and microRNA.org (http://www.microrna.org/). To validate the target mRNAs of miR-149, dual-luciferase reporter vectors containing the potential binding sites were constructed by amplifying the 3 '-UTR of the IL- $1 \alpha$, IL- $1 \beta$ and IL- 6 mRNAs and cloning them into the pGL3-reporter vector (obtained from the Department of Stem Cell and Regenerative Medicine). The primers used for IL-1 $\alpha$ 3'-UTR: Forward, GCTCTAGAGTGT TGACAGTTCATATGTAC and reverse, GCTCTAGATGTA ACATTATGGTCTGATC; primers used for IL-1 $\beta$ 3'-UTR: Forward, GCTCTAGACTGGACTTTCCTGTTG and reverse, GCTCTAGAGAAGTTTATTTCAGAACCATTG; primers 
Table I. Primers used for reverse transcription-quantitative polymerase chain reaction.

Gene Primer sequence

\begin{tabular}{ll}
\hline TGF- $\beta 1$ & F: GGAGCTGTACCAGAAATACAGC \\
TGF- $\beta 3$ & F: TGGCTGTTGAGAAGAGAGTCC \\
Collagen I & F: GAGTGGAGAGTACTGGATTGACC \\
Collagen III & F: ACAACAGGAAGCTGTTGAAGG \\
IL-1 $\alpha$ & F: GCTGCTGAAGGAGATGCC \\
IL-1 $\beta$ & F: GATGAAGTGCTCCTTCCAGG \\
IL-6 & F: GGATTCAATGAGGAGACTTGCC \\
NF-kB1 & F: CCAACAGATGGCCCATACCT \\
NF-kB2 & F: AAACAGCTGATGGCCCCTAC \\
p65 & F: TGTTCACAGACCTGGCATCC \\
RelB & F: GCTCTACTTGCTCTGCGACA \\
Rel & F: CCGGTGCGTATAACCCGTAT \\
GAPDH & F: AGAAGGCTGGGGCTCATTTG \\
U6 & F: GCTTCGGCAGCACATATACTAAAAT \\
miR-149 & TCTGGCTCCGTGTCTTCACTCCC
\end{tabular}

R: AACCCGTTGATGTCCACTTG

R: TGTCCACGCCTTTGAATTTG

R: GCCGCCATACTCGAACTG

R: GGCGAGTAGGAGCAGTTGG

R: ACGCCTGGTTTTCCAGTATC

R: GGAAAGAAGGTGCTCAGGTC

R: GGTTATTGCATCTAGATTCTTTGCC

R: AACCTTTGCTGGTCCCACAT

R: TGGCTGGTCCCTCGTAGTTA

R: GGGTACTCCATCAGCATGGG

R: CGGCGTCTTGAACACAATGG

R: CGGTTGTTGTCTGTGCTGTG

R: AGGGGCCATCCACAGTCTTC

R: CGCTTCACGAATTTGCGTGTCAT

TGF- $\beta$, transforming growth factor- $\beta$; GAPDH, glyceraldehyde-3-phosphate-dehydrogenase; IL, interleukin; NF, nuclear factor.

used for IL-6 3'-UTR: Forward, GCTCTAGACATTCCT TCTTCTGGTC and reverse, GCTCTAGATTTGAGGTAA GCCTACAC. The pRL-TK luciferase vector was used as an endogenous reference. HaCaT cells were co-transfected with the luciferase reporter plasmid $(1.2 \mu \mathrm{g})$ and pRL-TK $(0.12 \mu \mathrm{g})$, together with the miRNA mimic or a negative control using the Lipofectamine ${ }^{\circledR} 2000$ transfection reagent. Luciferase activity was analyzed $24 \mathrm{~h}$ after transfection using the Dual-Luciferase Reporter Assay System (Promega Corp., Madison, WI, USA) according to the manufacturer's instruction.

Western blot assay. To detect the protein levels regulated by miR-149 in fibroblasts, cells were washed with pre-chilled PBS, and the protein extracted with the Whole Cell Lysis Assay kit (Nanjing KeyGen Biotech Co., Ltd., Nanjing, China) according to the manufacturer's instruction. Protein concentration was measured using the BCA protein assay kit (Kaiji Biotechnology, Nanjing, China). Western blot analysis was performed according to standard protocols. Cell lysates containing loading buffer were boiled at $99^{\circ} \mathrm{C}$ for $5 \mathrm{~min}$, equal amounts of protein were run on $10 \% \mathrm{SDS}$ polyacrylamide gels, and transferred to a nitrocellulose membrane. After blocking in 5\% non-fat milk, membranes were incubated with 1:500 TGF- $\beta 1$ rabbit anti-human polyclonal antibody (Abcam, Cambridge, MA, USA), 1:500 TGF- $\beta 3$ mouse anti-human monoclonal antibody (Santa Cruz Biotechnology, Inc., Santa Cruz, CA, USA), 1:500 collagen I mouse anti-human monoclonal antibody (Abcam), and 1:1,000 collagen III mouse anti-human monoclonal antibody (Immunoway Biotechnology, Suzhou, China) for the detection of fibrosis, and 1:10,000 GAPDH mouse anti-human monoclonal antibody (ProteinTech Group, Inc., Chicago, IL, USA) for endogenous reference protein. After overnight incubation at $4^{\circ} \mathrm{C}$, the membranes were washed three times in PBST and incubated with an HRP-conjugated secondary antibody
(1:10,000; Beijing Dingguo Changsheng Biotechnology Co., Ltd., Beijing, China) for $1 \mathrm{~h}$ at $25^{\circ} \mathrm{C}$. Membranes were washed three times in PBST and immunoreactive bands were visualized by Amersham ECL Prime Western Blotting Detection Reagent (GE Healthcare, Piscataway, NJ, USA) using a Tanon 5200 detection system.

In vivo skin wound healing assay. Animal studies were performed in accordance with the guidelinges of the China Medical University Review Committee. Nine female Wistar rats were purchased from Beijing Weitong Lihua (Beijing, China). Animals were separately housed in $12 \mathrm{~h}$ light/dark cycles with ad libitum food and water. Twelve-week old animals weighing approximately $250 \mathrm{~g}$ were anesthetized by intra-peritoneal injection with pentobarbital sodium $(40 \mathrm{mg} / \mathrm{kg}$, Solarbio, China) and surgery was conducted under standard conditions. Two circular $12 \mathrm{~mm}$ full-thickness excisional wounds were created on the dorsal skin of each rat. HaCaT cells transfected with miR-149 mimic or a negative control were harvested from Nunc UpCell Surface dishes (Thermo Fisher Scientific, Inc., Waltham, MA, USA), which have a temperature responsive cell culture surface and enable harvest of cells with high vitality and intact surface proteins for cell transplantation. These cells were transplanted to the wound surface, followed by a coating with hydrogel (Hartmann AG, Heidenheim, Germany), transparent dressing, and medical gauze. Penicillin G sodium (80,000 units; Harbin Pharmaceutical Group Co., Ltd., Harbin, China) was used to prevent infection by intra-peritoneal injection. Animals were euthanized with overdose of pentobarbital sodium at days 1, 3 and 28 after wounding. Complete wound tissues of them were excised with a $2 \mathrm{~mm}$ margin around the wound edge. After fixation in $4 \%$ formaldehyde and dehydration in a $30 \%$ sucrose solution, tissues were embedded in Tissue-Tek O.C.T. compound (Sakura Fintek, Torrance, CA, USA) for frozen sections. Hematoxylin and eosin staining 
A

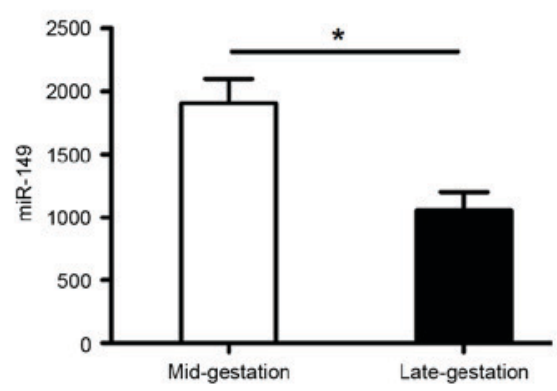

D

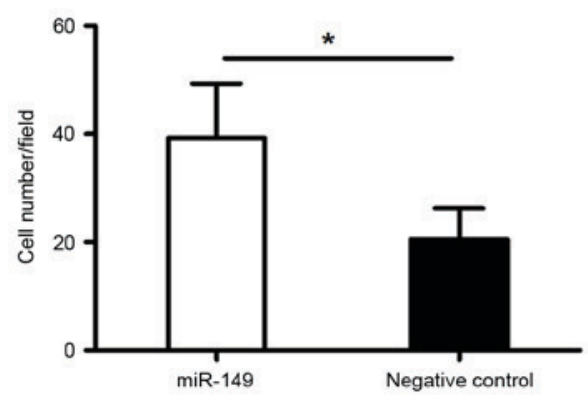

B

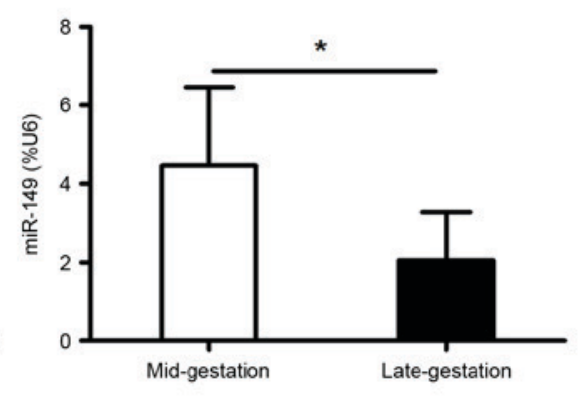

E

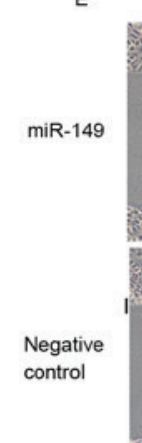

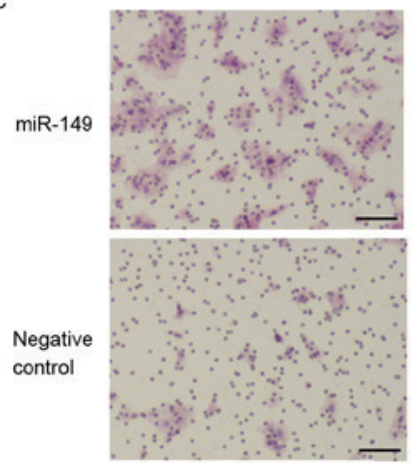

$24 \mathrm{~h}$

$36 \mathrm{~h}$

Figure 1. Expression of miR-149 in keratinocytes and role of miR-149 in HaCaT cells. Expression levels of miR-149 were detected by (A) next-generation sequencing and (B) Reverse transcription-quantitative polymerase chain reaction in fetal skin keratinocytes. The migration of transfected HaCaT cells was detected by $(\mathrm{C}$ and $\mathrm{D})$ transwell assay at $36 \mathrm{~h}$ and $(\mathrm{E})$ scratch assay at 0,24 , and $36 \mathrm{~h}$. ( $\mathrm{P}<0.05$, scale bars: $100 \mu \mathrm{m})$. miR, microRNA.

and Masson's trichrome staining (Solarbio, Beijing, China) were used for immunohistochemical analysis according to the manufacturer's instruction.

Statistical analysis. All results were performed at least in triplicate. Data shown were mean \pm standard deviation (SD). The differences were calculated by using a Student's t-test according to SPSS 16.0 software (SPSS, Inc., Chicago, IL, USA). $\mathrm{P}<0.05$ was considered to indicate a statistically significant difference.

\section{Results}

Characterization of miR-149 expression during mid-and late-gestational fetal skin keratinocytes. miR-149 was upregulated in the mid-gestational phase with a 1.85 -fold increase compared to that in the late-gestational phase (Fig. 1A). RT-qPCR was performed to validate this result (Fig. 1B).

miR-149 promotes migration of HaCaT cells. The migration of keratinocytes is one of the essential physiological process during skin wound healing. To evaluate whether miR-149 has an effect on HaCaT cell migration, we performed a transwell migration assay and an in vitro scratch assay. The results showed that overexpression of miR-149 accelerated $\mathrm{HaCaT}$ cell migration $48 \mathrm{~h}$ after transfection (Fig. 1C-E).

miR-149 targets $I L-1 \alpha, I L-1 \beta$, and IL- 6 genes in HaCaT cells. MicroRNAs participate in various biological processes by binding to their target mRNAs. Based on online predictions, we identified IL- $1 \alpha$, IL- $1 \beta$ and IL- 6 as the main target genes of miR-149 in HaCaT cells. We synthesized dual-luciferase reporter vectors containing the 3'-UTR of IL- $1 \alpha$, IL-1 $\beta$, and IL- 6 to investigate whether miR-149 binds to these areas to regulate the expression of IL- $1 \alpha$, IL-1 $\beta$, and IL-6 in HaCaT cells. The results showed (Fig. 2A) decreased luciferase activity in $\mathrm{HaCaT}$ cells after transfection with miR-149 mimic, as compared to those transfected with the negative control. Elisa and RT-qPCR results also showed that miR-149 suppressed both mRNA and protein expression of IL- $1 \alpha$, IL-1 $\beta$, and IL-6 in HaCaT cells transfected with miR-149 mimic, as compared to the negative control (Fig. 2B and C). Under inflammatory conditions, miR-149 also decreased the mRNA expression of IL- $1 \alpha$, IL-1 $\beta$, and IL-6 (Fig. 2D).

Regulation of miR-149 in $N F-\kappa B$ pathway. NF- $\kappa \mathrm{B}$ pathway can be activated by TNF- $\alpha$ through phosphorylating I $\mathrm{B}$, an inhibitor of NF- $\kappa \mathrm{B}$. Under inflammatory condition, RT-qPCR shows that miR-149 significantly decreased the expression of $\mathrm{NF}-\kappa \mathrm{B} 1, \mathrm{RelB}$, and Rel, which are subunits of NF- $\mathrm{NB}$ family (Fig. 2E).

Expression of TGF- $\beta 3$ and collagen III was upregulated at the $m R N A$ and protein level by miR-149 in fibroblasts. To further investigate how HaCaT cells transfected with miR-149 mimic affect the protein expression of dermal fibroblasts, we performed RT-qPCR and western blotting. HaCaT cells transfected with miR-149 mimic promoted the expression of TGF- $\beta 3$ and collagen III of fibroblasts, but had no significant effect on the expression of TGF- $\beta 1$ and collagen I (Fig. 3), as compared to the negative control. 


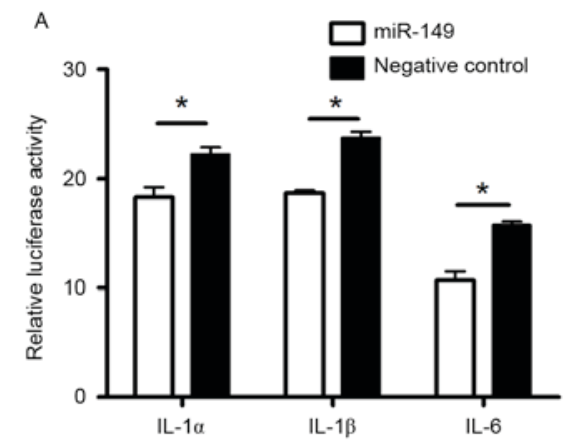

B

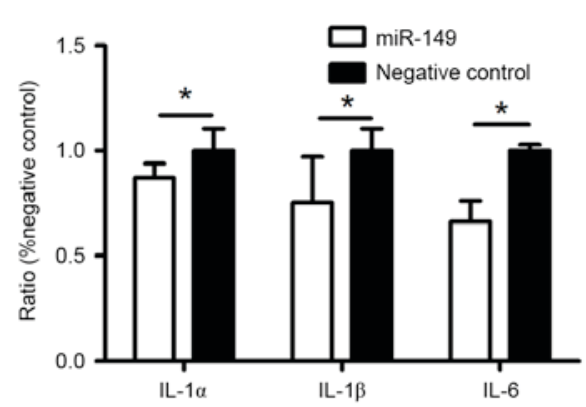

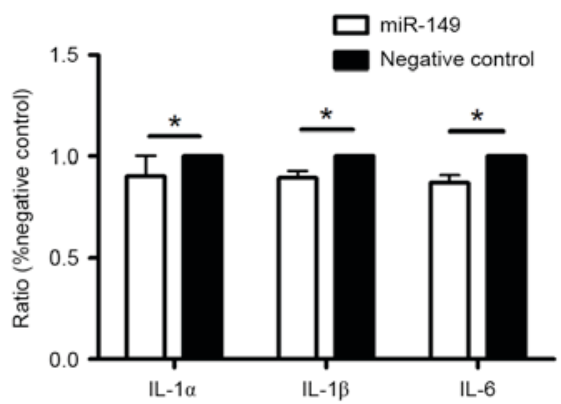
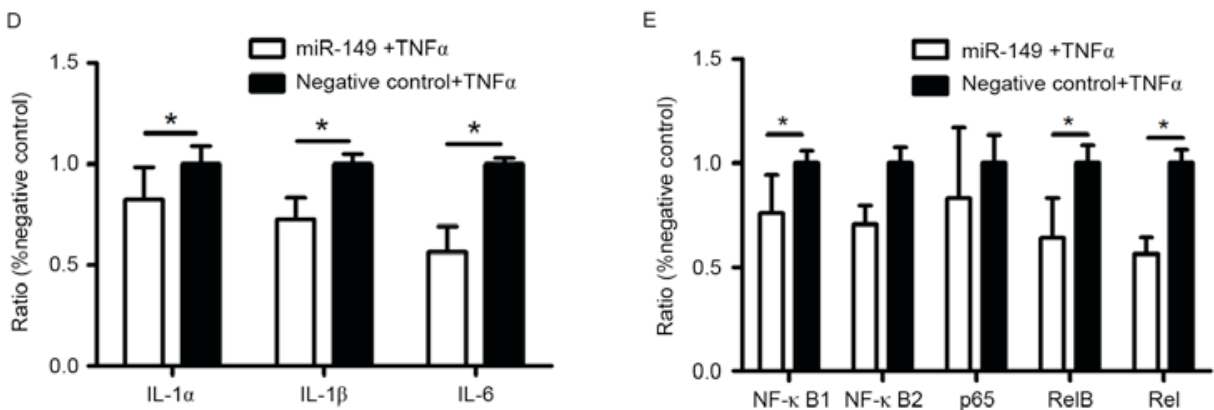

Figure 2. Anti-inflammatory role of miR-149 in HaCaT cells. HaCaT cells were transfected with luciferase reporter plasmids containing (A) IL-1 $\alpha$, IL-1 $\beta$, and IL-6 3'-UTR or pRL-TK plasmids together with miR-149 mimic or a negative control. Luciferase activity was measured 24 h after transfected. Expression of (B) IL-1 $\alpha$, IL-1 $\beta$, and IL-6 in supernatants was detected by ELISA after transfected with miR-149 mimic or a negative control 48 h. Expression of (C) mRNAs IL-1 $\alpha$, IL-1 $\beta$, and IL-6 in HaCaT cells was detected by RT-qPCR after transfected with miR-149 mimic or a negative control 48 h. Addition of TNF- $\alpha$ in HaCaT cells with transfected miR-149 mimic or a negative control was to reflect inflammatory condition, and the expression of (D) mRNAs IL-1 $\alpha$, IL-1 $\beta$, and IL-6 in TNF- $\alpha$-treated HaCaT cells was detected by RT-qPCR. Expression of (E) NF- $\mathrm{BB} 1, \mathrm{NF}-\kappa \mathrm{B} 2$, p65, RelB, and Rel in HaCaT cells was detected under the inflammatory condition by RT-qPCR. $(\mathrm{P}<0.05)$. RT-qPCR, reverse transcription-quantitative polymerase chain reaction; miR, microRNA; TGF- $\beta$, transforming growth factor- $\beta$; IL, interleukin; NF, nuclear factor.

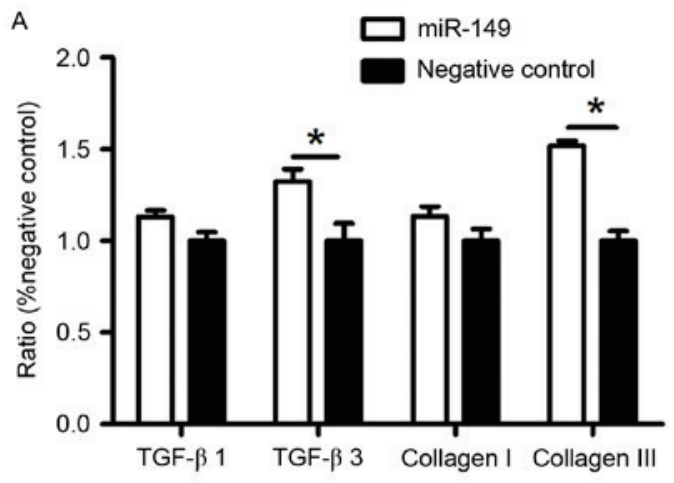

B

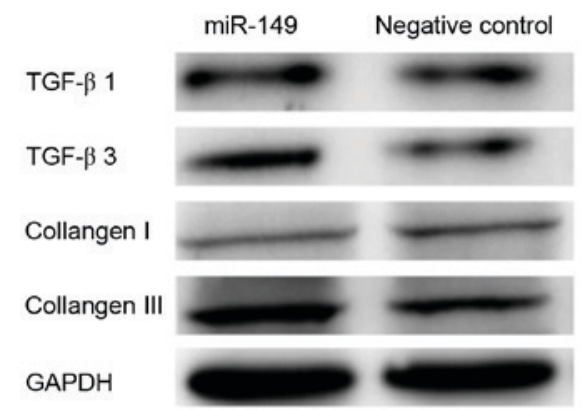

Figure 3. Regulation of miR-149 in fibroblasts under co-culture condition. Fibroblasts were co-cultured with HaCaT cells transfected with miR-149 mimic or a negative control, expression of TGF- $\beta 1$, TGF- $\beta 3$, collagen I, and collagen III in fibroblasts was detected by (A) reverse transcription-quantitative polymerase chain reaction and $(\mathrm{B})$ western blotting. $(\mathrm{P}<0.05)$. TGF- $\beta$, transforming growth factor- $\beta$; miR, microRNA.
HaCaT cells transfected with miR-149 mimic improve skin wound healing in a rat model. To determine whether miR-149 could indirectly improve skin wound healing through transfected $\mathrm{HaCaT}$ cells, we used a rat full-thickness skin excisional model (Fig. 4A). There were no obvious differences in these two groups in healing rate. The hematoxylin-eosin staining results showed that the wounds of the experimental group showed lower levels of inflammatory infiltration and more continuous epidermis and organized tissue, as compared to those in the negative control group (Fig. 4B and C). Masson's trichrome staining was used to assess the overall quality of the scarred areas and showed that collagen fibers within the wound areas of the experimental group were more compact and arranged, as compared to those in the negative control group (Fig. 4D).

\section{Discussion}

In this study, we focused on miR-149, which is upregulated in mid-gestational fetal skin keratinocytes, and studied its function in cutaneous wound healing in vitro and in vivo. Our results suggest that miR-149 restricts inflammation by downregulating the expression of IL- $1 \alpha$, IL- $1 \beta$, and IL- 6 , and promotes scarless wound healing by upregulating TGF- $\beta 3$ and collagen III in fibroblasts co-cultured with miR-149-transfected $\mathrm{HaCaT}$ cells. We believe that miR-149 may act as a positive regulator during the skin wound healing process. 
A

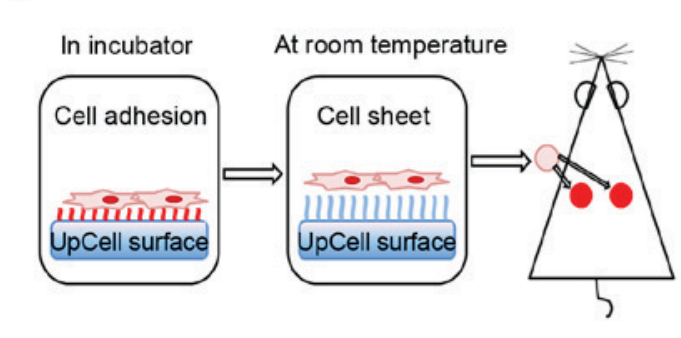

C

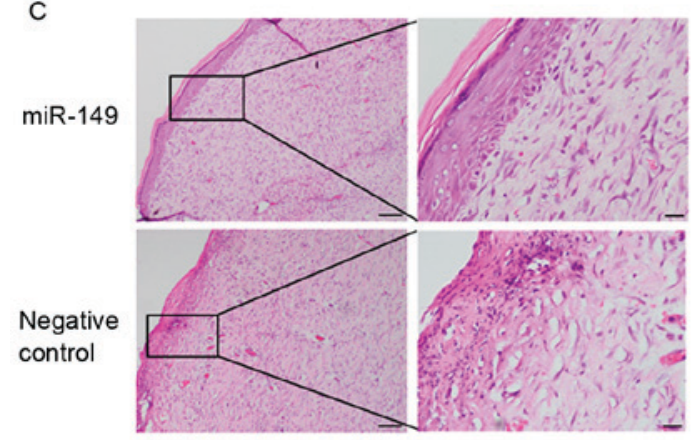

B

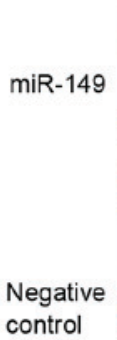

D

miR-149
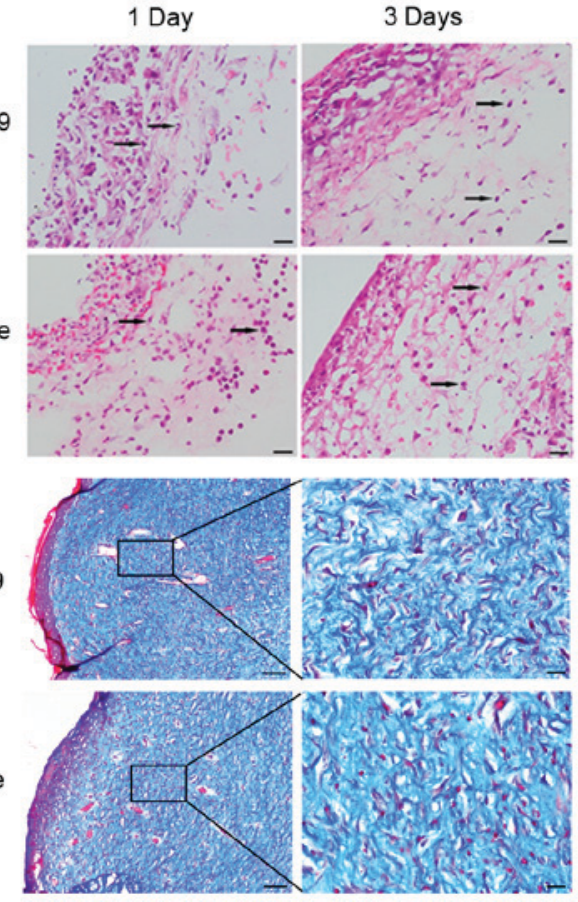

Figure 4. The role of miR-149 in the rat full-thickness excisional wound model. The experimental mode image was shown in (A). Hematoxylin and eosin staining (B) was used to detect infiltrating inflammatory cells (black arrows) at 1 day and 3 days after wounding, it shows that there were less infiltrating inflammatory cells in mimic-treated wounds than that in negative control-treated wounds (scale bars, $20 \mu \mathrm{m}$ ), and (C) more continuous epidermis and organized tissue at 28 days (scale bars, $100 \mu \mathrm{m}$ in left and $20 \mu \mathrm{m}$ in right). Masson's trichrome staining (D) shows that more arranged and reticular collagen arrangement in miR-149 mimic-treated wounds than that in a negative control-treated wounds at 28 days after wounding (scale bars, $100 \mu \mathrm{m}$ in left and $20 \mu \mathrm{m}$ in right). miR, microRNA.

It was first determined in the 1970s that fetal skin wounds can heal without the formation of scar tissue (21). Different from adult skin, fetal skin is associated with an attenuated inflammatory response during the wound healing process. Fetal skin wounds have lower expression of the pro-inflammatory cytokines IL-6 and IL-8 (22) and increased production of the anti-inflammatory cytokine IL-10 (23), in contrast to adult skin wounds. The reduction in inflammatory molecules can reduce scarring in postnatal wounds, thus, emphasizing the role of inflammation in scarless healing. Dermal fibroblasts are key effector cells during the skin wound healing process. Fetal fibroblasts are intrinsically different from adult fibroblasts in collagen synthesis, extracellular matrix production, and contractility (24). We performed small RNA sequencing analysis and uncovered the dynamic changes of miRNAs between mid- and late-gestational fetal skin keratinocytes. We first determined a group of miRNAs that targeted interleukins and were associated with the inflammatory response during skin wound healing. As reported, miR-149 is downregulated in osteoarthritis chondrocytes (15), in $24 \mathrm{~h}$ wounds relative to $0 \mathrm{~h}$ wounds (17), and in hypertrophic scar tissue (25). In this study, miR-149 was upregulated in mid-gestational fetal skin keratinocytes relative to late-gestational fetal skin keratinocytes. As mid-gestational fetal skin heals without scarring, we conclude that overexpression of miR-149 could contribute to scarless healing by dampening an excessive inflammatory response. But, miR-149 has no effect on skin wound healing rate.

Importantly, miR-149 can regulate the expression of IL-1 $\alpha$, IL-1 $\beta$, and IL-6 in HaCaT cells. Keratinocytes play an important role in innate immunity (26). Appropriate inflammation is critical in skin wound healing, but excessive and persistent inflammation may result in scar formation. In some studies, IL-1 $\beta$ and IL- 6 have been validated as target genes of miR-149 in chondrosarcoma cells (15) and a human embryonic kidney cell line $293 \mathrm{~T}$ (18). In this study, miR-149 suppressed the production of IL-1 $\alpha$, IL-1 $\beta$, and IL- 6 , which are essential pro-inflammatory cytokines in the inflammatory response. As reported, excessive infiltrating pro-inflammatory cytokines can lead to the formation of scar tissue. Our study suggests that miR-149 can suppress expression of pro-inflammatory cytokines IL-1 $\alpha$, IL-1 $\beta$, and IL- 6 both at basal levels and in inflammatory conditions, thus reducing scar tissue formation. To validate the pathway involving in the anti-inflammatory role of miR-149, should be involved in NF- $\mathrm{B}$ pathway, we used RT-qPCR to detect the expression of $N F-\kappa B$ family. Results showed that miR-149 can suppress expression of pro-inflammatory cytokines through $\mathrm{NF}-\kappa \mathrm{B}$ pathway. After degradation of phosphorylating I $\mathrm{B}$, activated dimer of $\mathrm{NF}-\kappa \mathrm{B}$ translocated into the nucleus and activated inflammatory pathway (27). The exact regulatory way of miR-149 needs more studies.

miR-149 can indirectly regulate gene expression of TGF- $\beta 3$ and collagen III in fibroblasts. Studies have shown that fetal skin wounds express higher levels of anti-fibrotic TGF- $\beta 3$ and decreased levels of pro-fibrotic TGF- $\beta 1$ and TGF- $\beta 2$ (24). The collagen deposited in the fetal skin wound is in a reticular pattern, unlike that in fibrotic tissues and hypertrophic scars, where collagen deposition appears as thick bundles parallel to the epidermal surface. Collagen III accounts for a higher ratio of the total collagen in fetal skin wounds than that in adult skin wounds and is regarded as a positive element in the 
healing process. Fibroblasts are the cells most involved in the synthesis and degradation of the extracellular matrix, and our results showed that TGF- $\beta 3$ and collagen III were upregulated in fibroblasts co-cultured with miR-149 mimic.

Skin wound healing is a complex biological process, thus, it may be difficult to improve markedly the quality of wound healing with a single factor. Our study suggests that miR-149 overexpression may reduce the expression of pro-inflammatory cytokines and improve the arrangement of collagen fibers. miR-149 may be a potential regulator in the skin wound healing process, though we are still researching other miRNAs that may have a synergistic effect with miR-149 to improve skin wound healing more effectively.

\section{Acknowledgements}

This study was supported by the Program for National Basic Research Program of China (no. 2012CB518103), the National Science Foundation of China (no. 81450017), the Shenyang Key Laboratory Project (no. F15-157-1-00), the Science and Technology Planning Project of Shenyang (no. F14-201-4-00), and the Science and Technology Planning Project of Liaoning Province (no. 2012225080).

\section{References}

1. Leavitt T, Hu MS, Marshall CD, Barnes LA, Lorenz HP and Longaker MT: Scarless wound healing: Finding the right cells and signals. Cell Tissue Res 365: 483-493, 2016.

2. Poetschke J and Gauglitz GG: Current options for the treatment of pathological scarring. J Dtsch Dermatol Ges 14: 467-477, 2016.

3. Wilgus TA, Ferreira AM, Oberyszyn TM, Bergdall VK and DiPietro LA: Regulation of scar formation by vascular endothelial growth factor. Lab Invest 88: 579-590, 2008.

4. Kryczka J and Boncela J: Leukocytes: The double-edged sword in fibrosis. Mediat Inflamm 2015: 652035, 2015.

5. Xu N, Meisgen F, Butler LM, Han G, Wang XJ, Söderberg-Nauclér C, Ståhle M, Pivarcsi A and Sonkoly E: MicroRNA-31 is overexpressed in psoriasis and modulates inflammatory cytokine and chemokine production in keratinocytes via targeting serine/threonine kinase 40. J Immunol 190: 678-688, 2013

6. Brant JO, Lopez MC, Baker HV, Barbazuk WB and Maden M: A comparative analysis of gene expression profiles during skin regeneration in mus and acomys. Plos One 10: e0142931, 2015.

7. Zhang L, Yan JW, Wang YJ, Wan YN, Wang BX, Tao JH, Chen B, Li BZ, Yang GJ and Wang J: Association of interleukin 1 family with systemic sclerosis. Inflammation 37: 1213-1220, 2014.

8. Werner S and Grose R: Regulation of wound healing by growth factors and cytokines. Physiol Rev 83: 835-870, 2003.

9. Takagi N, Kawakami K, Kanno E, Tanno H, Takeda A, Ishii K, Imai Y, Iwakura Y and Tachi M: IL-17A promotes neutrophilic inflammation and disturbs acute wound healing in skin. Exp Dermatol 26: 137-144, 2017.

10. Do DV, Ong CT, Khoo YT, Carbone A, Lim CP, Wang S, Mukhopadhyay A, Cao X, Cho DH, Wei XQ, et al: Interleukin-18 system plays an important role in keloid pathogenesis via epithelial-mesenchymal interactions. Brit J Dermatol 166: 1275-1288, 2012.
11. Yin H, Li X, Hu S, Liu T, Yuan B, Gu H, Ni Q, Zhang X and Zheng F: IL-33 accelerates cutaneous wound healing involved in upregulation of alternatively activated macrophages. Mol Immunol 56: 347-353, 2013

12. Lin L, Zhang YD, Chen ZY, Chen YX and Ren CP: The clinicopathological significance of miR-149 and PARP-2 in hepatocellular carcinoma and their roles in chemo/radiotherapy. Tumor Biol 37: 12339-12346, 2016.

13. Yang C, Sun C, Liang X, Xie S, Huang J and Li D: Integrative analysis of microRNA and mRNA expression profiles in non-small-cell lung cancer. Cancer Gene Ther 23: 90-97, 2016.

14. Pfeffer SR, Grossmann KF, Cassidy PB, Yang CH, Fan M, Kopelovich L, Leachman SA and Pfeffer LM: Detection of exosomal miRNAs in the plasma of melanoma patients. J Clin Med 4: 2012-2027, 2015.

15. Santini P, Politi L, Dalla Vedova P, Scandurra R and d'Abusco AS: The inflammatory circuitry of miR-149 as a pathological mechanism in osteoarthritis. Rheumatol Int 34: 711-716, 2014.

16. Palmieri D, Capponi S, Geroldi A, Mura M, Mandich P and Palombo D: TNFa induces the expression of genes associated with endothelial dysfunction through p38MAPK-mediated down-regulation of miR-149. Biochem Biophys Res Commun 443: 246-251, 2014.

17. Li DQ, Wang AX, Liu X, Meisgen F, Grünler J, Botusan IR, Narayanan S, Erikci E, Li X, Blomqvist L, et al: MicroRNA-132 enhances transition from inflammation to proliferation during wound healing. J Clin Invest 125: 3008-3026, 2015.

18. Xu G, Zhang Z, Xing Y, Wei J, Ge Z, Liu X, Zhang Y and Huang X: MicroRNA-149 negatively regulates TLR-triggered inflammatory response in macrophages by targeting MyD88. J Cell Biochem 115: 919-927, 2014.

19. Zhao F, Wang Z, Lang H, Liu X, Zhang D, Wang X, Zhang T, Wang R, Shi P and Pang X: Dynamic expression of novel miRNA candidates and miRNA-34 family members in early- to mid-gestational fetal keratinocytes contributes to scarless wound healing by targeting the TGF- $\beta$ pathway. Plos One 10: 0126087, 2015.

20. Liu XY, Wang Z, Wang R, Zhao F, Shi P, Jiang Y and Pang X: Direct comparison of the potency of human mesenchymal stem cells derived from amnion tissue, bone marrow and adipose tissue at inducing dermal fibroblast responses to cutaneous wounds. Int J Mol Med 31: 407-415, 2013.

21. Burrington JD: Wound healing in the fetal lamb. J Pediatr Surg 6: 523-528, 1971.

22. Zgheib C, Xu J and Liechty KW: Targeting inflammatory cytokines and extracellular matrix composition to promote wound regeneration. Adv Wound Care (New Rochelle) 3: 344-355, 2014.

23. Balaji S, King A, Marsh E, LeSaint M, Bhattacharya SS, Han N, Dhamija Y, Ranjan R, Le LD, Bollyky PL, et al: The role of interleukin-10 and hyaluronan in murine fetal fibroblast function in vitro: Implications for recapitulating fetal regenerative wound healing. Plos One 10: e0124302, 2015.

24. Larson BJ, Longaker MT and Lorenz HP: Scarless fetal wound healing: A basic science review. Plast Reconstr Surg 126: 1172-1180, 2010.

25. Li P, He QY, Luo CQ and Qian LY: Differentially expressed miRNAs in acute wound healing of the skin: A pilot study. Medicine (Baltimore) 94: e458, 2015.

26. Strbo N, Yin N and Stojadinovic O: Innate and adaptive immune responses in wound epithelialization. Adv Wound Care (New Rochelle) 3: 492-501, 2014

27. Pasparakis M: Regulation of tissue homeostasis by NF-kappaB signalling: Implications for inflammatory diseases. Nat Rev Immunol 9: 778-788, 2009. 